LETTERS

If you have a burning desire to respond to a paper published in $A D C$ or $F \& N$, why not make use of our "rapid response" option?

Log on to our website (www.archdischild. com), find the paper that interests you, click on "full text" and send your response by email by clicking on "submit a response".

Providing it isn't libellous or obscene, it will be posted within seven days. You can retrieve it by clicking on "read eletters" on our homepage.

The editors will decide, as before, whether to also publish it in a future paper issue.

\section{Late anaemia in Rh haemolytic disease}

As it is clearly stated in the review by Gottstein and Cooke, ${ }^{1}$ we consider it unethical to withhold or delay high dose intravenous immunglobulin (IVIG) treatment in infants with haemolytic disease of the newborn Since the study we did in 1995, ${ }^{2}$ we have treated 129 patients with Coomb's positive haemolytic disease of the newborn, with the same method and had to resort to exchange transfusions only in three cases. On the other hand, late anaemia is a frequent problem in these cases, necessitating multiple blood transfusions, with well known complications

The authors suggest that multiple doses of IVIG may reduce late anaemia. However, our observation in a limited number of cases is that, even multiple doses of IVIG are ineffective in preventing late anaemia. In an earlier unpublished study, we had shown that the erythropoietin levels were low in these infants. Therefore, we had conducted a double blind, randomised pilot study to investigate the effects of recombinant erythropoietin (rHEPO) in these patients. ${ }^{3}$ In this study, rHEPO was administered at a dose of 200 units $/ \mathrm{kg}$, subcutaneously, three times a week, starting at the 14th day of life and lasting for six weeks. This protocol reduced the number of erythrocyte transfusions significantly. With the impetus of this pilot study, we have used the same protocol for the subsequent 103 patients and the mean number of transfusions in this group was 1.5, with the majority of patients $(55 \%)$ not needing any transfusions at all. There were no complications, including changes in neutrophil or platelet counts, and haemorrhagic or infectious complications. The administration of rHEPO to patients with haemolytic disease of the newborn, who had received IVIG early in life, not only decreases the infants' exposure to multiple blood donors, but also diminishes the need for hospitalisation and hence the cost that is involved. Therefore, rHEPO treatment is a suitable alternative to erythrocyte transfusions in these infants.
F Ovalý

Istanbul Medical Faculty, Istanbul University, Turkey; fovali@yahoo.com

\section{References}

1 Gottstein R, Cooke RWI. Systematic review of intravenous immunoglobulin in haemolytic disease of the newborn. Arch Dis Child Fetal Neonatal Ed 2003;88:F6-F10

2 Dagoglu T, Ovalý F, Samancý N, et al. High dose intravenous immunoglobulin therapy for rhesus hemolytic disease. J Int Med Res 1995:23:264-71.

3 Ovalý F, Samancý N, Dagoglu T. Management of late anemia in rhesus hemolytic disease: Use of recombinant human erythropoietin (A pilot study). Pediatr Res 1996;39:831-4.

\section{Systematic review of intravenous immunoglobulin in haemolytic disease of the newborn}

We read with interest the recent review of Gottstein and Cooke. ${ }^{1}$ Their systematic review of trials reporting treatment of infants with proven $\mathrm{Rh}$ and/or $\mathrm{ABO}$ haemolytic disease of the newborn (HDN) treated with high dose intravenous immunoglobulin (HDIVIG) and phototherapy, with phototherapy alone demonstrated that significantly fewer infants required exchange transfusion in the HDIVIG group. The authors point out that anti-D is the commonest red cell antibody responsible for HDN. We have recently treated two children both of whom developed evidence of immune haemolysis due to anti-D antibodies acquired from IVIG.

The first patient, a 4 month old infant with Kawasaki's disease, was treated with intravenous immunoglobulin $(2 \mathrm{~g} / \mathrm{kg})$ with immediate control of fever and irritability. Ten days later her disease became clinically active again and she was therefore given a second dose of IVIG $(2 \mathrm{~g} / \mathrm{kg}$ from a different batch), which is a recognised therapeutic option. ${ }^{2}$ Her clinical condition again improved rapidly. A blood count two days after the second dose of IVIG showed that her haemoglobin had fallen suddenly by $2 \mathrm{~g} / \mathrm{dl}$ to $6.4 \mathrm{~g} / \mathrm{dl}$, the blood film showed spherocytes and the direct antiglobulin test was positive, evidence of immune haemolysis. Samples that were collected prior to the second dose of IVIG confirmed her blood group to be $\mathrm{AB} R \mathrm{Rh}$ positive with a negative direct antiglobulin test. Anti-D antibodies were now detected in the patient's serum; these were not present in her mother whose antibody screen was negative and whose blood group was A Rhesus D positive. The manufacturer of the IVIG investigated the batches used and reported that the IVIG used for the second dose contained anti-D. The second patient, a 12 year old boy with systemic juvenile idiopathic arthritis received a fifth dose of IVIG from the same batch. He was screened for evidence of haemolysis and his antiglobulin test was positive 14 days after treatment. He remained asymptomatic with no fall in haemoglobin.

IVIG is a pooled blood product not a drug; each batch is made from a pool of plasma collected from several thousand donors. Passive transfer of potentially significant red cell antibodies is a recognised hazard, reported in the company literature but only as a serological phenomenon, not as a clinical warning.
The first case is a reminder that such complications may have serious clinical consequences. We would agree with the comment of Gottstein and Cooke that the use of IVIG is not without potential risks, including haemolysis. IVIG is not universally effective in autoimmune haemolysis in older children and adults where steroids are the first choice.

Indications for the use of IVIG must be clear and evidence based, and as with all pooled blood products, including albumin solutions, the individual batch numbers must be recorded in the case notes, so that adverse events can be appropriately and fully investigated.

A G Cleary, B Brown, J Minards, J Sills Royal Liverpool Children's Hospital, Liverpool, UK; gavin.cleary@rlch-tr.nwest.nhs.uk

P Bolton-Maggs

Manchester Haemophilia Comprehensive Care Centre, Department of Clinical Haematology, Manchester Royal Infirmary, Oxford Road, Manchester M13 9WL, UK

\section{References}

1 Gottstein R, Cooke RWI. Systematic review of intravenous immunoglobulin in haemolytic disease of the newborn. Arch Dis Child Fetal Neonatal Ed 2003;88:F6-F10.

2 Dale R, Saleem M, Daw S, et al. Treatment of severe complicated Kawasaki disease with oral prednisolone and aspirin. J Pediatr 2000:137:723-6.

\section{High dose intravenous immunoglobulin in haemolytic disease of neonates}

It was encouraging to read article of Gottstein et $a l^{1}{ }^{1}$ on the use of high dose intravenous immunoglobulin (HDIVIG) in cases of haemolytic disease of newborns (HDN) with their conclusion showing the effectiveness of HDIVIG. I have the following observations to make with respect to implications on practice and future research.

Firstly, all the references mentioned were between three and ten years old. ${ }^{2-6}$ These trials did not take into consideration the irradiance of the phototherapy used, although they did observe the number of exchange transfusions performed. Presently, a combination of blue and white fluorescent light double surface phototherapy, with effective higher irradiances of $20-40 \mathrm{uW} / \mathrm{cm} 2 / \mathrm{nm}$, can practically eliminate the need for exchange transfusion, even in severe cases of HDN. Irradiance of phototherapy can be increased further by decreasing the distance between the phototherapy unit and the patient, especially with an undersurface phototherapy unit, keeping thermal and nursing issues under consideration.

Secondly, the authors did not address enterohepatic recirculation of bilirubin from the gut. Inexpensive measures can decrease the back entry of bilirubin from gut, like early enteral feeds, oral administration of agar agar, isbagol husk and so forth, and further reduce serum bilirubin levels. Further randomised controlled trials are required before administration of HDIVIG becomes routine in HDN. These trials should compare use of current effective phototherapy combinations with the 
highest possible irradiance, agents that decrease enterohepatic recirculation of bilirubin with or without HDIVIG, and the need for exchange transfusion in HDN. They should also address cost effectiveness and safety, considering the cost of HDIVIG in the developing world.

G Gupta

Armed forces Medical College, Pune, India; guptas-ip@eth.net

\section{References}

1 Gottstein R, Cooke RWI. Systematic review of intravenous immunoglobulin in haemolytic disease of the newborn. Arch Dis Child Fetal Neonatal Ed 2003;88:F6-F 10

2 Alpay F, Sarici SÜ, Okutan V, et al. High-dose intravenous immunoglobulin therapy in neonatal immune haemolytic jaundice. ActaPaediatr 1999;88:216-19.

3 Dagoglu T, Ovali F, Samaci N, et al. High-dose intravenous immunoglobulin therapy for rhesus haemolytic disease. J Int Med Res 1995; 23:264-71.

4 Rübo J, Albrecht K, Lasch P, et al. High-dose intravenous immune globulin therapy for hyperbilirubinemia caused by Rh hemolytic disease. J Pediatr 1992:121:93-7.

5 Voto LS, Sexer H, Ferreiro G, et al. Neonatal administration of high-dose intravenous immunoglobulin in rhesus hemolytic disease. J Perinat Med 1995; 23:443-51.

6 Rübo J, Wahn V, et al. Influence of high dosage immuno-globulin therapy on hyperbilirubinemia in rhesus hemolytic disease. A co-operative study. Monatsschr Kinderheilk 1996;144:516-19.

\section{Authors' reply}

We are grateful to our colleagues for their interest and responses to our paper. ${ }^{1}$ In response to Dr Ovalý's comments we agree that late anaemia can be a problem in babies who receive intravenous immunoglobulin (IVIG), as is also demonstrated in our systematic review. Even when infants have received exchange transfusions (XTs) top up red cell transfusions may be required. In a recent local audit of XTs, $35 \%$ of babies received top up red cell transfusions after one or more exchange transfusions. During a five year period from 1998-2002, 27 babies with Rhesus, Kell, or ABO incompatibility had 28 XTs. Gestation ranged from 28 to 40 completed weeks. Of 26 infants for whom follow up data was available, nine $(35 \%)$ had received top up red cell transfusions.

We read with interest Dr Ovalý and colleagues paper describing a double blind randomised controlled trial of subcutaneous recombinant human erythropoietin (rHEPO) and its use in this situation.

We await with interest the outcome of a Cochrane meta-analysis of this therapy in newborn infants (currently at the protocol stage).

We reviewed our computer database for a three year period from December 1999 to December 2002 to postulate what impact IVIG might have on our population of babies with haemolytic disease of the newborn. Two hundred and five babies had a positive direct Coombs test (DCT) result. Of these infants, 12 received XTs. There is a degree of under ascertainment with this database as there were four additional babies who required an XT during this time period. However, we make the assumption that the proportions of those missed requiring XTs is similar to the proportions of DCT positive babies who were missed from the database. Eighty five babies had moderate or strongly positive DCT. Of these 11 received an XT, giving an XT rate in this group of $13 \%$. After IVIG the relative risk of requiring an XT is $0.28,{ }^{1}$ thus with IVIG the XT rate would be reduced to $3.6 \%$, decreasing the number of XTs to three and therefore preventing eight. If IVIG were administered to all babies with moderate or strongly positive DCT, in our population the number needed to treat would be 10.6 to prevent one XT. The degree of positivity of the DCT is an objective validated assessment of the strength of antigen/antibody reaction, determined by the degree of agglutination in the laboratory. ${ }^{3}$ During the three year period of this database there was only one infant who had only a weakly positive DCT and required an XT.

We were interested to read Dr Cleary and colleagues case reports. We recognise that IVIG is not specific for a particular type of haemolysis and that it is a pooled blood product. We therefore agree that all the usual procedures regarding documentation of batch number and so on are followed as for any other blood product. IVIG has been used previously even in preterm and low birthweight infants ${ }^{4}$ and is currently being used in the INIS Trial. ${ }^{5}$ As with any drug or blood product we will need to remain vigilant for the occurrence of any adverse events.

R Gottstein, RWI Cooke Neonatal Unit, Liverpool Women's Hospital, Liverpool L8 7SS, UK; rgottstein@ntlworld.com

\section{References}

1 Gottstein R, Cooke RWI. Systematic review of intravenous immunoglobulin in haemolytic disease of the newborn. Arch Dis Child Fetal Neonatal Ed 2003;88:F6-10.

2 Ovali F, Samanci N, Dagoglu T.

Management of late anemia in rhesus hemolytic disease: use of recombinant human erythropoietin (a pilot study). Pediatr Res 1996:39:831-4.

3 Dunsford I, Bowley CC. Techniques in blood grouping, 2nd edn, vol II. Edinburgh: Oliver and Boyd, 1967:270, 287.

4 Ohlsson A, Lacy JB. Intravenous immunoglobulin for preventing infection in preterm and/or low birthweight infants. (Cochrane Review). The Cochrane Library Oxford: Update Software, 2000:issue 3.

5 http://www.npeu.ox.ac.uk/Inis.htm

\section{Discharging twins separately from neonatal units}

We recently had a debate in our unit about whether or not it was better for a well twin to remain with its sibling in hospital until the latter was fit to be discharged. Our current practice is to keep the well twin in the special care baby unit until its twin is fit for discharge. The group who favoured separate discharge cited reduced risk of nosocomial infection, decreased costs, cot availability, and the possibility of settling into a routine with one twin at home as supportive factors for their argument. Those against separate discharge cited impaired bonding, breast feeding difficulties, and transport issues as their reasons.

We took the discussion to the RCPCH and NICU-net email discussion groups and found no clear consensus. Our American colleagues routinely send multiples home separately and cite health insurance companies as a major factor in this decision. They find little problem with this arrangement. European opinion was split between the two camps. British doctors seemed to be in favour of asking the parents opinion, so we identified 10 sets of twins from the last three years who could have been sent home separately. We then sent their parents a questionnaire exploring their opinions; five (50\%) were returned.

Most parents agreed that their twins were ready for discharge at different times and said that they would have preferred separate discharge. However, they believed that they had been given this option and had not taken it. They realise that this would have caused problems with visiting, feeding, and bonding with the remaining twin even although they all had their own transport. They did not think that having one twin home first would have helped them to adjust and settle into a routine. Their preferred option would have been to have roomed in with the well twin while the other twin stayed on the special care baby unit.

Our current practice is that we have an informed discussion with the parents when this situation arises. As one email respondent (a doctor and father of twins) wrote "Bringing up twins is full of decisions about when to pair them and when to split them up."

$$
\begin{array}{r}
\text { J P Chapman, K Lange } \\
\text { James Paget Hospital, Lowestoft Road, Gorleston, } \\
\text { Norfolk NR31 6LA, UK } \\
\text { Correspondence to: Dr Chapman; } \\
\text { john.chapman@ipaget.nhs.uk }
\end{array}
$$

\section{Role of serum peak levels of vancomycin in neonatal intensive care units}

We would like to comment on the article by Tan et al. ${ }^{1}$ The purpose of measuring serum levels of a drug is either to monitor the toxicity of the drug or the therapeutic concentration for a particular condition. Emergence of infections with $\beta$-lactam-resistant Staphylococcus epidermidis, Staphylococcus aureus, and Enterococcus $\mathrm{sp}$, has led to the frequent use of vancomycin in neonates. Vancomycin has historically had a reputation for toxicity. Many of its original adverse reactions, including ototoxicity and nephrotoxicity, were probably due impurities in the formulation. ${ }^{2}$ Now that a more purified form is available, these adverse reactions are uncommon. However, concomitant administration with aminoglycosides or other nephrotoxins may increase the risk of toxicity. ${ }^{3}$ Effective drug therapy is measured by response, not by achievement of a particular circulating drug concentration. Because the association between vancomycin peak concentrations and toxicity is poor, some have recommended measuring trough concentration only ${ }^{4}$ as this study is clearly documenting, but others have suggested not measuring any concentrations in the majority of children with normal renal function. ${ }^{5}$ However, in critically ill premature neonates with poor glomerular filtration rate, prematurity, and compromised cardiovascular function, it remains prudent to measure both peak and trough concentrations in those with poor or changing renal function. Caution must be exercised when other nephrotoxic or ototoxic drugs such as aminoglycisides are administered concurrently. ${ }^{6}$ In this study, the authors did not mentioned the concomittent use of aminoglycoside.

S Jain

Southern Illinois University School of Medicine, Springfield Illinois, USA; jainsunil@hotmail.com

\section{References}

1 Tan W-H, Brown N, Kelsall A W, et al. Dose regimen for vancomycin not needing serum 
peak levels? Arch Dis Child Fetal Neonatal Ed 2002;87:F214-6.

2 Baillie GR, Neal D. Vancomycin ototoxicity and nephrotoxicity. A review. Med Toxicol Adv Drug Exp 1988;3:376-86.

3 Rybak MJ, Albrecht LM, Boike SC, et al. Nephrotoxicity of vancomycin, alone and with an aminoglycoside. J Antimicrob Chemother 1990;25:679-87.

4 Saunders NJ. Why monitoring peak vancomycin concentration? Lancet 1994;344: 1748-50.

5 Thomas MP, Steele RW. Monitoring serum vancomycin concentrations in children. Is it necessary? Pediatr Infect Dis J

1998;17:351-2.

6 Moellering RC, Krogstad DJ, Greenblatt DJ Vancomycin therapy in patients with impaired renal function: a nomogram for dosage. Ann Intern Med 1981;94:343-6.

\section{Methaemoglobinaemia with concurrent blood isolation of Saccharomyces and Candida}

Saccharomyces boulardii is closely related to Saccharomyces cerevisiae and is used as biotherapeutic agent, ${ }^{1}$ although some reports sugges pathogenicity. ${ }^{2}$ We present a case of neonatal fungaemia with concurrent methaemoglobinaemia, occurring after a brief period of treatment with $S$ boulardii. A male infant was born at 30 weeks of gestational age by caesarean section because of intrauterine growth re striction and maternal hypertension. The baby was well apart from persistent gastrointestinal symptoms that hampered feeding and forced parenteral support. During the third week of life, administration of $S$ boulardit (Codex DNB; half a capsule a day, equivalen to $2.5 \times 10^{9}$ organisms) was started in an attempt to prevent bacterial overgrowth. After four days of treatment, the baby developed symptoms suggesting sepsis and an unex plained methaemoglobinaemia (methaemoglobin concentration $=16 \%$ ). Codex was stopped and empirical antibiotic coverage, including liposomal amphotericin B, was started. Blood cultures showed growth of Candida albicans, but the central venous cath eter tip was negative. Methaemoglobin levels halved in two days $(7 \%)$, but remained constant during the following two weeks of antifungal treatment. Blood cultures at that point showed growth of $S$ cerevisiae, which is susceptible to amphotericin B, in the absence of any clinical finding; again, the catheter tip was sterile. Methaemoglobin concentration was still abnormal (6.2\%). Liposomal amphotericin B treatment was prolonged for a further six days and then discontinued. At this time, methaemoglobin levels were near normal $(3 \%)$, and blood cultures were negative. The gastrointestinal symptoms resolved with age and full gastrointestinal function was achieved.

Recovery of Saccharomyces two weeks after administration had been stopped suggests persistence in the gut. It is tempting to think that the methaemoglobinaemia was caused by the continued presence of the yeasts, perhaps through excessive host production of nitric oxide. Several studies have shown that nitric oxide plays a pivotal role in the interaction between yeasts and the phagocytic system, ${ }^{3}$ and it is well known that this radical readily oxidises haemoglobin. It is also reasonable to link late bloodstream invasion by Saccharomyces to previous enteric mucosal damage caused by a Candida infection, which was itself probably gut related. The recovery of $S$ cerevisiae in place of $S$ boulardii has been reported by others, ${ }^{4}$ and can be explained by the similarities between the two. It is ironic that the intervention used to prevent sepsis from enteric overgrowth not only did not succeed but was itself a cause of the problem that it was intended to prevent.

M S Lungarotti, D Mezzetti, M Radicioni Policlinico Monteluce, Via Brunamonti, Perugia, PG 06100, Italy; dm2@unipg.i

\section{References}

1 McCullough MJ, Clemons KV, McCusker JH, et al. Species identification and virulence attributes of Saccharomyces boulardii (nom. inval.). J Clin Microbiol 1998;36:2613-17.

2 Ipson MA, Blanco CL. Saccharomyces cerevisiae sepsis in a 35-week-old premature infant. A case report. J Perinatol 2001;21:459-60.

3 Netea MG, Meer JW, Verschueren I, et al. CD40/CD40 ligand interactions in the host defense against disseminated Candida albicans infection: the role of macrophage-derived nitric oxide. Eur Immunol 2002;32:1455-63.

4 Bassetti S, Frei R, Zimmerli W. Fungemia with Saccharomyces cerevisiae after treatment with Saccharomyces boulardii. Am J Med 1998; 105:71-2.

\section{Wafting does work}

We were interested to see the article "Oxygen administration in infants", ${ }^{1}$ and eLetter responses. The original article and $e$ Letters were unsure of the efficacy of "non-contact" oxygen delivery or "wafting" as it is more commonly known. Our study "The efficacy of noncontact oxygen delivery methods", ${ }^{2}$ demonstrated how effective wafting oxygen can be. We found that an area of $34 \mathrm{~cm}$ by $37 \mathrm{~cm}$ obtained a concentration of $>30 \%$ when oxygen is delivered by face mask at $10 \mathrm{l}$ /minute. Although this is not a substitution for the more reliable methods of administration as detailed by Drs Frey and Shann, in the short term it can be used with confidence.

We caution against holding a self inflating resuscitation bag over an infant's airway (without manipulation of the bag itself), as it delivers a negligible amount of oxygen. It is much more efficient to use the oxygen tubing without any attachments.

P Davies NETS, Sydney, Australia; daviespatrick@hotmail.com

D Cheng

Department of Oncology, Great Ormond Street Hospital, London, UK

A Fox

Department of Paediatric Allergy, St Mary's Hospital, London, UK

L Lee

Neonatal Intensive Care Unit, Rosie Maternity Hospital, Cambridge, UK

\section{References}

1 Oxygen administration in infants. B Frey and F Shann. Arch Dis Child Fetal Neonatal Ed 2003:88:F84-F88.

2 Davies P, Cheng D, Fox A, et al. The Efficacy Of Noncontact Oxygen Delivery Methods. Pediatrics. 2002;110:964-7.

\section{CORRECTION}

We wish to apologise for an error that occurred in a letter by Daniels et al (Arch Dis Child Fetal Neonatal Ed 2003;88:F257). The first line of the third paragraph should have read: The salient results were that two thirds of granulomas resolved over a three week period without cauterisation. 